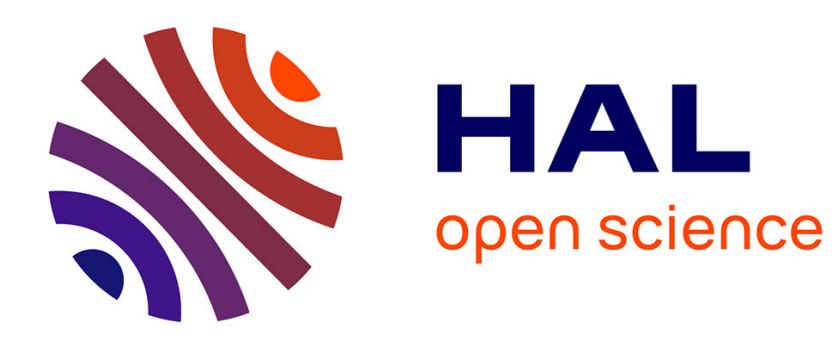

\title{
Les vaccins vétérinaires de nouvelle génération
}

J.M. Aynaud

\section{- To cite this version:}

J.M. Aynaud. Les vaccins vétérinaires de nouvelle génération. Productions Animales, 1991, 4 (1), pp.89-95. hal-00895928

\section{HAL Id: hal-00895928 \\ https://hal.science/hal-00895928}

Submitted on 1 Jan 1991

HAL is a multi-disciplinary open access archive for the deposit and dissemination of scientific research documents, whether they are published or not. The documents may come from teaching and research institutions in France or abroad, or from public or private research centers.
L'archive ouverte pluridisciplinaire HAL, est destinée au dépôt et à la diffusion de documents scientifiques de niveau recherche, publiés ou non, émanant des établissements d'enseignement et de recherche français ou étrangers, des laboratoires publics ou privés. 
INRA Prod, Anim.,

1991, 4 (1), 89 - 95

\section{J.M. AYNAUD}

INRA Département de Pathologie Animale 37380 Nouzilly

\section{Les vaccins vétérinaires de nouvelle génération}

\section{L'objectif de la vaccination de l'animal domestique de rente est essentiellement la réduction des pertes économiques causées aux productions animales par les maladies infectieuses et parasitaires. Dans un environnement à risque pour les troupeaux, la vaccination contribue directement à maintenir l'intégrité des fonctions de l'organisme intervenant dans la production de viande, de lait et d'œufs. Les vaccins vétérinaires constituent un outil privilégié pour la prévention des maladies des animaux domestiques qu'ils soient de rente ou de compagnie.}

Les premiers vaccins vétérinaires ont été mis au point il y a déjà plus d'un siècle par Louis Pasteur qui, en hommage à Jenner (1798), a créé les concepts de vaccin et de vaccination. Avec l'aide des vétérinaires Galtier et Chauveau de l'Ecole vétérinaire de Lyon, Bouley, Nocard et Delafond de l'Ecole vétérinaire d'Alfort et enfin Toussaint de l'Ecole vétérinaire de Toulouse, Louis Pasteur s'est intéressé à la mise au point de vaccins contre le choléra des poules (1880), la fièvre charbonneuse du mouton (1880), la péripneumonie des bovins (1882) et enfin le rouget du porc (1883). A la faveur de ces travaux menés dans un délai de 3 ans, Pasteur a jeté les bases scientifiques à partir desquelles tous les vaccins ultérieurs ont été préparés jusqu'au milieu des années 50. Peu de temps après Pasteur, les bases scientifiques de l'immunité humorale et de l'immunité cellulaire étaient respectivement découvertes par Von Behring et Paul Erlich, et par Elie Metchikoff.

\section{Résumé}

Les vaccins vétérinaires doivent non seulement être efficaces et totalement inoffensifs, mais répondre à des contraintes économiques strictes imposées par les éleveurs en amont des filières productrices de viande, de lait et d'ceufs. Le contrôle des maladies virales et bactériennes dévastatrices telles la fièvre aphteuse, la peste porcine et la brucellose a été réalisé grâce à des vaccins conventionnels dont la plupart sont encore largement utilisés dans le monde. Les nouvelles technologies issues de la fusion cellulaire et de la recombinaison génétique offrent la possibilité d'éliminer certains inconvénients qui peuvent subsister dans les préparations actuelles.

L'un des atouts majeurs des vaccins de la nouvelle génération sera la possibilité de différencier animaux infectés et vaccinés.
C'est un vétérinaire, Gaston Ramon, qui, à l'Institut Pasteur, a découvert en 1923 le principe des anatoxines ainsi que celui des adjuvants de l'immunité. Enfin, aux Pays-Bas en 1952, Frenkel mit au point la méthode pour préparer le premier vaccin à virus inactivé contre la fièvre aphteuse, produit à partir de virus cultivé sur l'épithélium lingual de bovin maintenu en survie. La méthode Frenkel fut le départ de la vaccinologie vétérinaire, nouvelle technologie industrielle qui prit son essor à Lyon (Institut Français de la Fièvre aphteuse) et diffusa ensuite dans de nombreux pays pour faire face aux terribles épizooties de fièvre aphteuse qui ravagaient alors périodiquement les populations de bovins, d'ovins, de caprins et de porcins. Récemment, en 1989, la Faculté vétérinaire de l'Université d'Utrecht (Pays-Bas) a créé, pour la première fois en Europe, une Chaire pour l'enseignement de la vaccinologie.

Le développement des technologies nouvelles dérivées de la fusion cellulaire et de la recombinaison d'ADN a entraîné toute une série d'innovations dans les vaccins vétérinaires au niveau de leur conception, de leur préparation et de leur utilisation.

L'objectif de cet article est d'examiner, de façon critique, l'évolution des concepts et des approches qui ont conduit aux vaccins actuels, ou qui, à partir des technologies nouvelles, vont conduire à court terme aux vaccins dits de nouvelle génération et à des méthodes de vaccination plus performantes pour l'animal domestique de rente qu'il soit un mammifère, un oiseau ou un poisson. 


\section{Contraintes propres aux vaccins vétérinaires}

Les vaccins destinés à l'animal domestique de rente souffrent de nombreuses contraintes dont ne font pas l'objet les vaccins destinés à l'animal domestique de compagnie, ou à l'espèce humaine.

L'utilisateur de vaccins vétérinaires au sein d'une filière de production animale (filière porc, filière lait, etc.) considère ces derniers comme un des moyens d'améliorer la productivité de son élevage en réduisant les pertes dues aux maladies infectieuses (viroses, bactérioses, mycoplasmoses, chlamydioses) et parasitaires (fasciolose, helminthoses, coccidioses, hypodermose). Les qualités exigées par un tel utilisateur sont les suivantes :

- coût minimum acceptable de la dose vaccinale

- interventions réduites au minimum, compte tenu du manque croissant de main d'cuvre disponible dans les élevages à caractère industriel

- présentation et administration parfaitement adaptées aux conditions actuelles de l'élevage

- totale innocuité à court et long terme

- efficacité en terme de protection.

Le contrôleur officiel du Ministère de l'Agriculture, sur qui repose toute la garantie d'un bon vaccin pour l'utilisateur, exige les qualités suivantes pour accorder l'autorisation de mise sur le marché :

- innocuité et efficacité conformes aux réglementations en vigueur au niveau national et au niveau de la CEE

- stabilité génétique des vaccins vivants

- respect des normes pour la protection de l'environnement (vaccins vivants recombinants)

- détection des animaux vaccinés des animaux infectés par un test sérologique de routine.

Pour l'industriel de la vaccinologie vétérinaire, la mise au point de bons vaccins possédant l'ensemble des qualités exigées par les différents partenaires en production animale est une opération qui demande du temps (5 à 10 ans en moyenne entre l'idée et la mise sur le marché), beaucoup de matière grise et des investissements lourds en laboratoires et en installations expérimentales "étanches» pour les animaux, indispensables pour mettre en œuvre les tests de "challenge " pour l'évaluation du pouvoir protecteur des vaccins.

Pour l'industriel, la rentabilité financière d'une telle opération est souvent faible, compte tenu des contraintes d'ordre économique ou réglementaire. Le marché du médicament vétérinaire est en effet étroit (moins de $5 \%$ du marché du médicament humain). De plus, à l'intérieur de ce marché, celui des vaccins vétérinaires est éclaté en plusieurs sous-secteurs liés à la multiplicité des espèces animales de rente (5 de mammifères, 5 d'oiseaux, poissons : salmoniculture) et aussi à la diversité des agents pathogènes responsables de maladies dans chacune de ces espèces. L'aide financière n'existe pas et, de ce fait, le coût unitaire de la dose vaccinale doit être nécessairement très faible (autour d'un centime pour le poulet, quelques francs pour un porc) compte tenu des marges bénéficiaires des éleveurs, qui sont de plus en plus étroites. Dans certains cas (coccidioses des poulets), les vaccins antiparasitaires potentiels vont se trouver en compétition avec les molécules antiparasitaires introduites dans les aliments distribués aux animaux. Il faut, par ailleurs, rappeler les nombreuses contraintes tenant au développement de réglementations restrictives imposées par les comités d'éthique concernant les conditions des essais sur le terrain des vaccins recombinants issus du génie génétique.

Cependant, par rapport aux vaccins destinés à l'homme, les vaccins vétérinaires bénéficient d'un avantage considérable concernant les conditions de l'évaluation de l'innocuité et de l'efficacité. Ces deux qualités vaccinales sont en effet obligatoirement contrôlées directement sur l'espèce animale à laquelle le vaccin est destiné et ceci sur des lots importants d'animaux homogènes et standardisés. Cet avantage constitue pour les utilisateurs une excellente garantie de qualité.

Tableau 1. De lidée au laboratoire à la seringue dans l'étable: les 10 étapes successives de la mise au point d'un vaccin vétérinaire.

1 - Le vaccin est-il nécessaire?

2 - Choix d'une approche technologique appropriée.

3 - Estimation de la faisabilité, compte tenu des différentes contraintes.

4 - Recherche de l'antigène à potentialité vaccinale.

5 - Préparation d'un immunogène prototype.

6 - Mise au point du vaccin et contrôle des qualités exigées.

7 - Mise au point des conditions de la production industrielle.

8 - Mise au point de la méthode de vaccination adaptée aux conditions de la pratique (voie, dose, calendrier).

9 - Essais sur le terrain.

10 - Demande d'autorisation de mise sur le marché (AMM).

Enfin, donnée importante, la durée de l'utilisation d'un vaccin vétérinaire est d'autant plus brève que le vaccin s'est avéré efficace en termes de protection et de réduction des pertes économiques.

Pour le chercheur situé en amont, la mise au point de vaccins est, en soi, une finalité de recherches, mais le plus souvent c'est une retombée majeure du progrès des connaissances et des technologies concernant les propriétés in vitro (biologie moléculaire, antigénicité) et in vivo (réponse immunitaire de l'ani- 
mal hôte) des agents pathogènes responsables des maladies.

La mise au point de bons vaccins vétérinaires est largement conditionnée par les progrès accomplis au niveau des concepts et des technologies dans les différentes disciplines telles que la virologie, la microbiologie, la parasitologie, l'immunochimie et l'immunologie.

\section{Des vaccins vétérinaires : pourquoi, pour protéger contre quoi et comment?}

Si elle permet de contrôler efficacement les maladies, la vaccination n'empêche souvent pas les agents pathogènes de continuer à persister et à circuler à bas bruit dans la population animale. Aussi, le contrôle d'une maladie par la vaccination est considérée comme la première étape du processus d'éradication. Ultérieurement, quand la maladie a disparu d'un territoire du fait de l'efficacité de la vaccination, l'interdiction de cette dernière est une mesure nécessaire pour des raisons sanitaires et surtout commerciales. C'est, par exemple, le cas en France, comme au Royaume-Uni, de la Peste porcine et de la Fièvre aphteuse.

La protection vaccinale de l'animal contre des maladies virales ou bactériennes où l'immunité est de caractère plutôt systémique (Fièvre aphteuse, Peste porcine, Peste bovine, Rouget du porc, Maladie d'Aujeszky du porc, Rhinotrachéite infectieuse des bovins, etc.) a été obtenue relativement facilement en raison de la bonne efficacité des vaccins disponibles. En revanche, de nombreuses difficultés subsistent pour induire une protection immunitaire de niveau égal contre les maladies où l'immunité est essentiellement locale (entérites, mammites). Il n'existe pas encore de bons vaccins capables de protéger efficacement la glande mammaire de la vache laitière contre les infections bactériennes. Par ailleurs, certaines maladies virales à caractère systémique ne bénéficient pas encore de solution satisfaisante en matière de vaccins, et ceci, bien que les connaissances des propriétés de l'agent pathogène responsable ou des mécanismes immunitaires aient progressé de façon spectaculaire ces dernières années ; c'est le cas de toutes les rétroviroses animales (leucose bovine, leucoses aviaires, rétroviroses du mouton et de la chèvre, anémie infectieuse du cheval, rétroviroses félines), de la maladie de Marek des poulets et de la Peste porcine africaine.

Tout reste à faire pour les nouvelles maladies apparues très récemment; c'est le cas de la maladie hémorragique du lapin, qui est un fléau pour les élevages et dont on n'a pas encore réussi à cultiver le virus responsable.

Enfin, les maladies parasitaires qui restent encore des pathologies préoccupantes en Europe (coccidioses aviaires, fasciolose et hypodermose des ruminants) et, le plus souvent, dominantes dans les zones tropicales des pays en développement (trypanosomiases), sont plus ou moins bien contrôlées par des molé- cules antiparasitaires dont certaines sont soupçonnées avoir des effets indésirables sur la faune sauvage (arthropodes) de l'environnement. De bons vaccins antiparasitaires restent donc à mettre au point. Ils pourraient constituer une alternative intéressante aux molécules antiparasitaires. Mais de grandes difficultés subsistent tenant à la complexité de l'antigénicité du parasite vis-à-vis duquel la réponse immunitaire de l'animal hôte est souvent désarmée (échappement du parasite aux défenses immunitaires de l'organisme infesté).

Depuis une dizaine d'années, avec le développement des biotechnologies, nous assistons à la multiplication des approches technologiques proposées pour la mise au point de vaccins. De ce fait, les chercheurs sont confrontés au choix de la meilleure approche, compte tenu d'une part de l'état des connaissances concernant l'agent pathogène et de la réponse immunitaire de l'animal hôte et d'autre part des exigences des utilisateurs potentiels dans la filière de production animale concernée. Car disposer de nouveaux immunogènes plus performants ne représenterait qu'un progrès limité sans la mise au point de nouveaux modes de présentation et d'administration qui viseront à accroître l'efficacité des nouveaux vaccins et à simplifier les programmes de vaccination, surtout quand il s'agit de stimuler, par exemple, l'immunité locale du tractus digestif. Les problèmes pratiques posés par la vaccination orale du renard contre la rage ou celle de la truie contre la gastroentérite transmissible en sont une bonne illustration.

\section{Les vaccins vétérinaires actuels}

C'est à Louis Pasteur que l'on doit la démonstration que l'on peut transformer des microorganismes pathogènes en vaccins par atténuation de leur virulence. A partir de cette voie, la sélection orientée de mutants ayant perdu en partie ou en totalité leur virulence (par passages en série sur l'animal de laboratoire tel que le lapin par exemple, ou par passages aveugles dans des conditions particulières en culture cellulaire) a constitué pendant longtemps une approche performante qui a permis de préparer de nombreus vaccins viraux vivants efficaces. C'est ainsi que les vaccins actuels contre la maladie d'Aujeszky (souche "Alfort-26"), contre la Peste porcine classique (souche "Thiverval ») ou contre la Chlamydiose abortive (souche «INRA-1B ») ont été préparés dans les laboratoires de l'INRA à partir de mutants «froids » obtenus en culture cellulaire à basse température par rapport à $37-38^{\circ} \mathrm{C}$. C'est grâce à ce type de vaccins, qualifiés maintenant de conventionnels, que les grandes maladies virales dévastatrices ont été peu à peu éliminées de notre territoire. Le vaccin contre la Salmonellose abortive des ruminants est constitué par un mutant particulier de Salmonella abortus bovis (travaux à l'INRA de Tours). L'inactivation de l'agent pathogène (vaccin actuel contre l'entérite virale à coronavirus des veaux (travaux à l'INRA de Jouy-en-Josas) ou de ses toxines (vaccin contre les mammites des ruminants (travaux à l'INRA de Tours), ainsi que la
Les vaccins conventionnels sont constitués le plus souvent de microorganismes atténués ou inactivés. Ils ne permettent pas de différencier, par un test sérologique de routine, les animaux infectés des animaux vaccinés puisqu'ils induisent chez ces derniers la synthèse des mêmes anticorps. 
préparation d'antigènes protecteurs (vaccin contre l'entérite colibacillaire du veau (travaux à l'INRA de Theix) continuent d'apporter des solutions efficaces contre ces maladies animales en attendant que les vaccins de nouvelle génération issus des biotechnologies, (immunochimie, recombinaison génétique) soient réellement opérationnels sur le terrain et puissent prendre le relais des vaccins actuels. Rappelons que les inconvénients majeurs des vaccins actuels, dits conventionnels sur le plan de l'éradication, tiennent principalement au fait qu'il n'est pas possible de différencier par un test sérologique les animaux vaccinés des animaux infectés.

\section{Les vaccins vétérinaires de nouvelle génération}

\section{1 / Les nouveaux concepts}

La connaissance des bases moléculaires de la virulence et de l'immunogénicité a conduit à un progrès considérable dans la mise au point de vaccins. En effet, le génie génétique a offert la possibilité de séparer les antigènes (épitopes) susceptibles d'induire la réponse immunitaire protectrice (principe vaccinant) du reste des constituants du microorganisme pathogène. Dans ces conditions, certains vaccins vivants recombinants ne renferment plus de particules pathogènes puisque la partie du génome impliquée dans la virulence (gènes TK et gI pour le virus Aujeszky) a été éliminée par délétion. Seuls les gènes impliqués dans l'immunogénicité ont été conservés dans la construction finale. De même, certains vaccins non vivants ne renferment plus que le ou les motifs antigéniques responsables de l'immunogénicité auxquels ont été "greffées » des molécules à effet adjuvant ou facilitant la présentation de l'antigène aux lymphocytes. Généralement, le « principe vaccinant " appartient à l'une des protéines structurales de l'agent pathogène. Mais il peut être également constitué par des composants non structuraux (protéines NS-1 des flavirus, protéases sécrétées par certains parasites tels que le varron : l'hypodermine A) ou même par des protéines non codées par l'agent pathogène tels que des récepteurs cellulaires spécifiques ou des antiidiotypes.

Le sérum des animaux ainsi immunisés est alors dépourvu d'anticorps vis-à-vis de certains constituants du microorganisme pathogène, alors qu'en revanche le sérum des animaux ayant fait l'objet de l'infection naturelle en est pourvu. On dispose alors d'un moyen élégant de différencier, par un test de laboratoire de routine basée sur une réaction sérologique, les animaux infectés des animaux vaccinés. Cela permet de mener de front vaccination et éradication. Une telle approche est maintenant opératonnelle pour la Maladie d'Aujeszky du porc. Des projets sont en cours pour d'autres maladies (Peste porcine, Gastroentérine transmissible, Brucellose...).

La possibilité de différencier les animaux vaccinés des animaux infectés constitue, de toute évidence, le progrès actuel le plus mar-
Tableau 2. Les 3 grands types de vaccins issus du génie génétique.

A - Vaccins recombinants sous-unités à partir de la production de l'antigène par des systèmes d'expression tels que des bactéries, des levures ou des cellules animales après infection avec un virus recombinant (baculovirus et cellules d'insectes, poxvirus avec les cellules VERO ou les cellules BHK).

B - Vaccins vivants recombinants à partir de la construction de souches virales ou bactériennes hybrides (« chimères") exprimant l'antigène lors de leur multiplication ou à leur surface (greffe moléculaire d'un motif antigénique).

C - Vaccins vivants atténués par manipulation génétique: création de nouvelles souches virales ou bactériennes ayant perdu leur virulence par délétion ou par mutagenèse dirigée.

quant pour les vaccins vétérinaires, car il va permettre d'améliorer considérablement la stratégie globale pour l'éradication des maladies infectieuses et parasitaires des animaux domestiques.

\section{2 / Technologies à l'origine des approches pour les vaccins de nouvelle génération}

- Les réactifs : l'arsenal d'enzymes de restriction (endonucléases) et d'enzymes de modification (polymérase, ligase) a permis de réaliser une véritable chirurgie moléculaire du patrimoine génétique. L'arsenal des anticorps monoclonaux a permis de mettre en place l'immunochimie pour la caractérisation des motifs antigéniques « minimum» susceptibles d'être responsables de l'immunogénicité vaccinale.

- Les technologies :

- l'ensemble des techniques d'intervention sur l'ADN : le clonage, le séquençage et la recombinaison in vitro (insertion, délétion, substitution, construction) ont atteint un haut niveau de perfectionnement. En particulier la construction de bactéries "déguisées" au moyen de la technique génétique de greffe moléculaire de motifs antigéniques sélectionnés venant d'un microorganisme pathogène sur une protéine structurale (Lamb, Pili) exprimée à la surface externe d'une bactérie "véhicule » inoffensive comme $E$. coli ou Salmonella est une approche prometteuse permettant d'aborder par exemple les problèmes de présentation d'antigènes lors de la vaccination orale.

- la synthèse peptidique et l'immunochimie.

- les molécules adjuvantes de l'immunité et les complexes immunostimulants (ISCOM).

- Le matériel animal et cellulaire :

- les animaux de types génétiques particuliers (histocompatibilité, mutants, sensibilité ou résistance à un agent pathogène ou à une maladie). 
- les animaux à haut statut sanitaire: statut "germ-free ", IOPS (indemne d'organismes pathogènes spécifiques). L'éradication des rétrovirus endogènes dans des lignées de poulets a été entrepris récemment à l'INRA de Tours.

- lignées cellulaires ayant des caractéristiques et des fonctions particulières : cellules « ES" (Embryonic Stem), cellules helper transcomplémentantes, lymphocytes pérennisés et hybridomes.

\section{3 / Les nouvelles stratégies : les différents types de futurs vaccins issus du génie génétique}

Les vaccins vivants recombinants (vaccins " chimères») sont constitués par des souches virales ou bactériennes atténuées hybrides exprimant un antigène vaccinal étranger lors de leur multiplication ou à leur surface.

exemples : - le virus de la vaccine comportant un gène d'enveloppe du virus rabbique (travaux menés par Transgène, Rhone-Mérieux, le CNEVA et l'Université de Liège).

- l'adénovirus humain type 5 comportant le gène d'enveloppe gp 50 du virus de la maladie d'Aujeszky du porc (travaux menés par l'Ecole vétérinaire d'Alfort et le CNRS).

- le virus de la rhinotrachéite infectieuse bovine comportant le gène VP1 du virus de la fièbre aphteuse (travaux menés au Texas).

- l'herpesvirus de la maladie d'Aujeszky comportant un gène d'enveloppe du virus de la Peste porcine (travaux menés à Lelystad aux Pays-Bas).

Tableau 3. Vecteurs utilisés pour la construction de vaccins vivants recombinants.
Virus
Poxvirus : virus de la vaccine variole du canard variole aviaire.
Herpesvirus : Aujeszky du porc bovine.
Autres : ?
Bactéries
Escherichia coli
Salmonella typhimurium.

Adénovirus humain, canin, simien.

Rhinotrachéite infectieuse

Retrovirus : retrovirus aviaires défectifs.

Les vaccins vivants atténués par manipulation génétique sont constitués de nouvelles souches virales ou bactériennes ayant perdu leur virulence par mutagenèse dirigée ou par délétion.

exemple : la souche atténuée (TK-) du virus de la maladie d'Aujeszky ayant perdu par délétion un ou plusieurs gènes dont celui de la thymidine kinase TK (travaux aux USA).
Les vaccins recombinants sous unitaires sont constitués par de l'antigène vaccinal produit en abondance par des systèmes d'expression appropriés mis au point par recombinaison génétique.

exemple: l'antigène gp50 de l'enveloppe du virus de la maladie d'Aujeszky produit par des cellules d'insectes infectées par le baculovirus recombinant comportant le gène gp50 (travaux en cours au CNEVA en collaboration avec l'INRA).

Les vaccins synthétiques sont constitués par la séquence de la portion de la protéine candidate virtuellement immunogène, qui a été synthétisée. Un tel vaccin chimiquement défini est conceptuellement idéal.

exemple : l'oligopeptide correspondant aux aminoacides $141-160$ et 200-213 de la protéine VP-1 du virus de la fièvre aphteuse confère une relative protection à l'animal dans les conditions expérimentales (travaux menés au Royaume-Uni).

Les vaccins anti-idiotypes sont constitués par les antigènes non codés par l'agent pathogène. Il s'agit d'anticorps anti-idiotypes qui représentent l'image interne du motif antigénique de départ codé par l'agent pathogène.

Les vaccins anti-récepteurs sont constitués par les antigènes du récepteur spécifique pour l'attachement de virus pathogènes à la surface des cellules de l'hôte.

La vaccination génétique consiste à créer des lignées d'animaux résistant à une maladie, en transférant, au niveau de la lignée germinale des individus reproducteurs, un ou plusieurs gènes connus pour intervenir dans la résistance à cette maladie, ou des séquences complémentaires à celle du génome de l'agent pathogène. exemple: gènes du système d'histocompatibilité du poulet intervenant dans la résistance de certaines lignées d'animaux aux tumeurs induites par les virus de la maladie de Marek ou par les rétrovirus des leucoses aviaires (travaux en cours à l'INRA de Tours en collaboration avec l'Université de Lyon et le Collège de France).

\section{4 / Avantages et difficultés des vaccins vétérinaires de nouvelle génération}

Les vaccins synthétiques présentent des avantages certains (composition chimique définie et stable d'un lot à l'autre, stabilité à la température ambiante et innocuité totale en particulier vis-à-vis des risques de contaminants). Cependant, des difficultés majeures limitent les espoirs de leur développement en médecine vétérinaire :

- le choix de la molécule porteuse, de l'adjuvant et de l'agent de couplage chimique ;

- la non applicabilité aux épitopes discontinus ; - la variabilité de la réponse post-vaccinale en fonction de l'haplotype du système majeur d'histocompatibilité de l'individu vacciné ;

- l'efficacité, médiocre en terme de protection, nécessite plusieurs rappels.

Les vaccins anti-idiotypes et anti-récepteurs constituent conceptuellement des alternatives
L'anticorps induit à partir de l'agent pathogène est lui-même un antigène qui est susceptible d'induire la fabrication d'anticorps de structure voisine de l'antigène de départ. Les vaccins anti-idiotypes sont constitués par ces anticorps. 
Tableau 4. Approches technologiques ayant abouti aux vaccins actuels dits "conventionnels" ou "de première génération " ou susceptibles d'aboutir aux vaccins de nouvelle génération.

\section{Vaccins Conventionnels}

$\begin{array}{ll}\text { vaccin vivant } \longleftarrow \begin{array}{l}\text { sélection de } \\ \text { mutténts atténués }\end{array} & \\ \text { atté }\end{array}$ l'agent pathogène ou de ses produits (toxines)

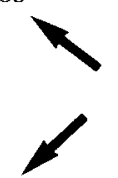

Agent Pathogène (virus, bactérie, parasite)
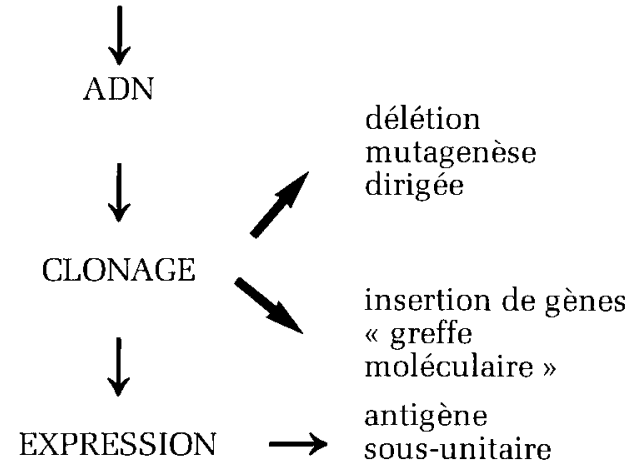

sous-unitaire délétion mutagenèse dirigée

insertion de gènes

" greffe

antigène

\section{Vaccins de nouvelle génération}

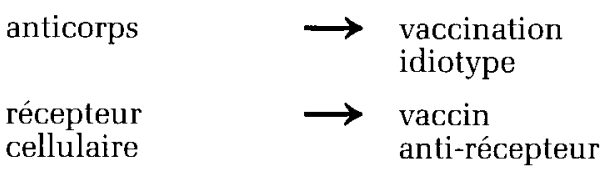
$\longrightarrow$ vaccin vivant atténué par manipulation génétique

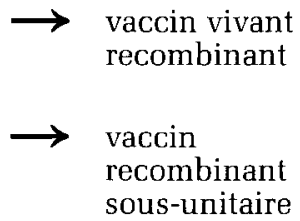

tout à fait attractives mais, en terme de protection immunitaire, les résultats publiés à ce jour n'ont pas encore satisfait les espoirs placés dans ces deux approches originales.

Les vaccins vivants vont vraisemblablement constituer le champ d'application majeur des nouvelles technologies issues du génie génétique compte tenu des sévères contraintes spécifiques à la médecine vétérinaire et cela pour les trois raisons suivantes:

- l'immunité conférée est de longue durée, à la fois cellulaire et humorale, à la fois locale et générale ;

- une faible charge antigénique et une seule administration de vaccin sont nécessaires, donc le coût est modéré ;

- les adjuvants de l'immunité ne sont pas nécessaires.

Ce sont de telles raisons qui sont à l'origine de l'immense succès de la vaccination antivariolique dans le monde pour l'espèce humaine avec en plus une grande facilité d'administration, une excellente stabilité du vaccin à la température ambiante et un très faible coût de revient de la dose vaccinale.

Cependant les vaccins vivants recombinants, « chimères » ou non, devront être examinés et contrôlés avec les mêmes critères que ceux utilisés actuellement pour les vaccins dits conventionnels en ce qui concerne la pathogénicité résiduelle et le risque de reversion vers la virulence à la faveur de mutations et surtout de recombinaisons génétiques in vivo au sein des populations animales chez qui d'autres microorganismes sont susceptibles de circuler à bas bruit (autres souches vaccinales recombinantes) ou de persister à l'état latent (rétrovirus endogènes, herpesvirus].

\section{Conclusion}

Le champ des approches possibles pour les vaccins vétérinaires s'est considérablement élargé suite au développement des nouvelles technologies issues du génie génétique. Même si les efforts entrepris n'ont pas tous encore abouti à des applications pratiques conduisant à des nouveaux vaccins utilisés sur le terrain à grande échelle, il est certain qu'ils ont tous largement contribué à l'acquisition de connaissances fondamentales sur les agents pathogènes et sur les mécanismes immunitaires de la réponse de l'animal hôte à l'agression virale, microbienne ou parasitaire.

Il ressort, en définitive, que l'un des atouts majeurs des vaccins vétérinaires de nouvelle génération sera la possibilité de différencier les individus infectés des individus vaccinés. L'enjeu est considérable car il devient alors possible de merner de front des campagnes de vaccination et des opérations d'éradication face aux enzooties.

\section{Références bibliographiques}

Cette liste ne présente volontairement que des articles de synthèse récents.

AYNAUD J.M., LAUDE H., 1988. Les vaccins vétérinaires. Biofutur, $\mathrm{n}^{t \prime}$ 69, 65-70.

BITTLE J.L., MURPHY F.A., 1989. Vaccine biotechnology. Advances in Vétérinary Science and Comparative Medicine. 33, 1-429.

BLOOM B.R., 1989. Vaccines for the third world. Nature, 342, 115-120.

BONA C.A., 1987. Les vaccins du futur. La recherche. 18 , $672-682$ 
BROWN F., 1988. Use of peptides for immunization against foot and mouth disease. Vaccine. 6, 180-182.

FIELDS B.N., CHANOCK R.M., 1989. What biotechnology has to offer vaccine development. Reviews of infectious diseases. 11, sup 3, 519-529.

GAMBLE H.R., MURELL K.D., 1987. Progress in the development of vaccines against parasitic diseases. Immunology Letters. 16, 329-336.

GIRARD M., 1990. Les vaccins 100 ans après Louis Pasteur. Ann. Institut Pasteur. Actualités, 102-113.

HENDERSON L.M., KATZ J.B., ERICKSON G.A., MAYFIELD J.E., 1990. In vivo and in vitro genetic recombination between conventional and gene-deleted vaccine strains of pseudorabies. Ann. J. Vet. Res., 51, 1656-1662. HOFNUNG M., 1990. Des bactéries « déguisées » pour une nouvelle génération de vaccins. Ann. Institut Pasteur. Actualités, 114-119.

KATZ J.B., HENDERSON L.M., ERICKSON G.A., 1990 Recombination in vivo of pseudorabies vaccine strains to produce new virus strain. Vaccine. 8, 286-288.

KATZ J.B., 1990. Genetically engineered vaccines for control of Aujeszky's disease (pseudorabies). Vaccine. 8 , 420-424.
LAUDE H., 1988. Perspectives d'utilisation des biotechnologies dans la lutte contre les maladies infectieuses du porc. J. Rech. Porc en France, 20, 133-140.

MELNICK J.L., 1989. Virus vaccines : principles and prospects. Bull OMS, 67, 105-112.

Mise au point de vaccins - nouvelles approches : memorandum d'une réunion de l'OMS. Bull. OMS, 1985, 63, 851-857.

MOLITOR M., THAWLEY D., 1987. Pseudorabies vaccines : past, present, and future. Compendium Food Animal, 9, 409-416.

PELLERIN J.L. 1988. Vaccins et outils de diagnostic: actualités et perspectives. Rev. Med. Vet., 139, 127-162.

Potential use of live viral and bacterial vectors for vaccines. WHO meeting. Vaccine, 1990, 8, 425-437.

STEWART M.W., HOWARD C.R., 1987. Synthetic peptides: a next generation of vaccines. Immunology today. 8, 51-57.

ZANETTI M., SERCARZ E., SALK J., 1987. The immunology of new generation vaccines. Immunology today. 8 , 18-25.

\section{Summary}

\section{Veterinary vaccines of new generation.}

Veterinary vaccine must not be harmless and highy efficient but they have to take into account of the severe economical constraints imposed by breeders upstream of network for meat, milk and eggs. Control of viral and bacterial diseases such foot and mouth disease, swine fever and brucellosis, was got with conventional vaccines the most of which are still widely used in the world. New technologies resulting of cell fusion and genetic recombination offer ability to eliminate some disadvantages of conventional vaccines. Possibility to differentiate vaccinated animals from infected will be the major advantage of new generations vaccines.

AYNAUD J.M., 1991. Les vaccins vétérinaires de nouvelle génération. INRA Prod. Anim., 4 (1), 89-95. 